\title{
Field Research and Numerical CFD Analysis of Humpback Whale-Inspired Shortboard Fins ${ }^{+}$
}

\author{
David Shormann ${ }^{1,2}$, Marc in het Panhuis ${ }^{2,3}$ and Luca Oggiano ${ }^{2,4, *}$ \\ 1 DIVE, LLC, Haleiwa, HI 96712, USA; drshormann@gmail.com \\ 2 Surf Engineering Association, Kiama Downs 2533, NSW, Australia; panhuis@uow.edu.au \\ 3 Surf Flex Lab, Australian Institute for Innovative Materials, University of Wollongong, Wollongong \\ 2500, NSW, Australia \\ 4 Norwegian University of Science and Technology-SIAT (Senter for Idrettsanlegg og Teknologi), K. \\ Hejes vei 2b, 7042 Trondheim, Norway \\ * Correspondence: luca.oggiano@ntnu.no \\ + Presented at the 13th conference of the International Sports Engineering Association, Online, \\ 22-26 June 2020.
}

Published: 15 June 2020

\begin{abstract}
Compared to other Olympic sports, little research exists on competitive shortboard surfing-especially research comparing field and numerical data. In this paper, GPS sensors with 9-axis motion sensors were used to collect data on nearly 2000 surfed waves. Data were collected from four surfers of differing skill levels, ranging from intermediate/advanced (Level 6) to topranked professional (Level 9). The results revealed a positive correlation between surfer skill level and roll/pitch/yaw rates during a cutback. Some surfers used two different fin types: a standard commercial fin (C), and a 3D-printed, humpback whale-inspired fin (RW). Statistically significant cutback performance improvements were seen when surfers used the RW fin. Because of the skill level differences suggested by the field data, dynamic computational fluid dynamics (CFD) analysis was performed to simulate cutback maneuvers at three different rotation rates (roll/pitch/yaw). Sustained resultant forces relative to the rider direction were lower for RW fins during the turn, suggesting a less-skilled surfer could generate faster and more powerful turns using RW fins. Field results also confirmed that a skill Level 8 surfer performed closer to skill Level 9 when using RW fins, but not control fins. Surfers experienced more stability using RW fins, and CFD results confirmed RW's ability to dampen the effects of turbulent flow.
\end{abstract}

Keywords: shortboard fin performance; STAR-CCM+, CFD; GPS tracking; humpback whale; tubercled leading edge; cutback maneuver; passive flow control; biomimetics

\section{Introduction}

Surfing is a global sport that involves catching and riding waves on a surfboard fitted with fins. Currently, only one other study compares field performance and numerical (computational fluid dynamics, CFD) results of different surfboard fin designs [1]. The study involved a single, longboard-style surfing fin, comparing a standard longboard fin to a tubercled, "real whale" (RW) design. Static CFD results showing improved efficiency and an expanded operating envelope for RW led to field testing of a prototype RW design. Results from over 650 surfed waves, comparing RW to a standard longboard fin confirmed the CFD results, with significant improvements in max speed, average speed, and distance surfed on individual waves.

Rather than single longboard fins, the present study compares field and numerical results from 3-fin thruster sets. Introduced by Simon Anderson in 1980 [2], thruster sets are commonly used in high performance shortboard surfing, where maneuverability and control are key 
performance factors. Specifically, this paper focuses on the cutback, or top turn (Figure S1), an important maneuver during recreational and competitive surfing [3-5]. To gain more understanding of field performance of RW vs. control fins attached to shortboards, this study uses dynamic CFD to simulate field results and compare forces imparted to 3-fin thruster sets.

\section{Materials and Methods}

\subsection{Field Research}

Field research materials and methods are discussed in more detail elsewhere $[1,5,6]$. The research involved surfers of 4 different skill levels as defined in Reference [7], including intermediate (Level 6), expert (Levels 7 and 8) and WCT, or World Surf League Men's World Championship Tour (Level 9). Data was collected using a commercial tracking system with a 10 $\mathrm{Hz}$ sampling rate (TraceUp, USA). Attached on the nose of the surfboard, the device contained nine inertial sensors and a GPS, and was used to monitor and quantify performance from almost 2,000 surfed waves. Data for the WCT surfer was provided by TraceUp. For this experiment, RW refers to "real whale" fins with tubercled leading edges and other passive flow control features found on the humpback's unique pectoral fins. Control (C) refers to any set of thruster fins with a straight leading edge. Figure 1 shows a surfboard and images of $C$ and RW thruster sets, and Table S1 provides detailed information on participants, surfboards and fins. Table S2 lists performance means by skill level.
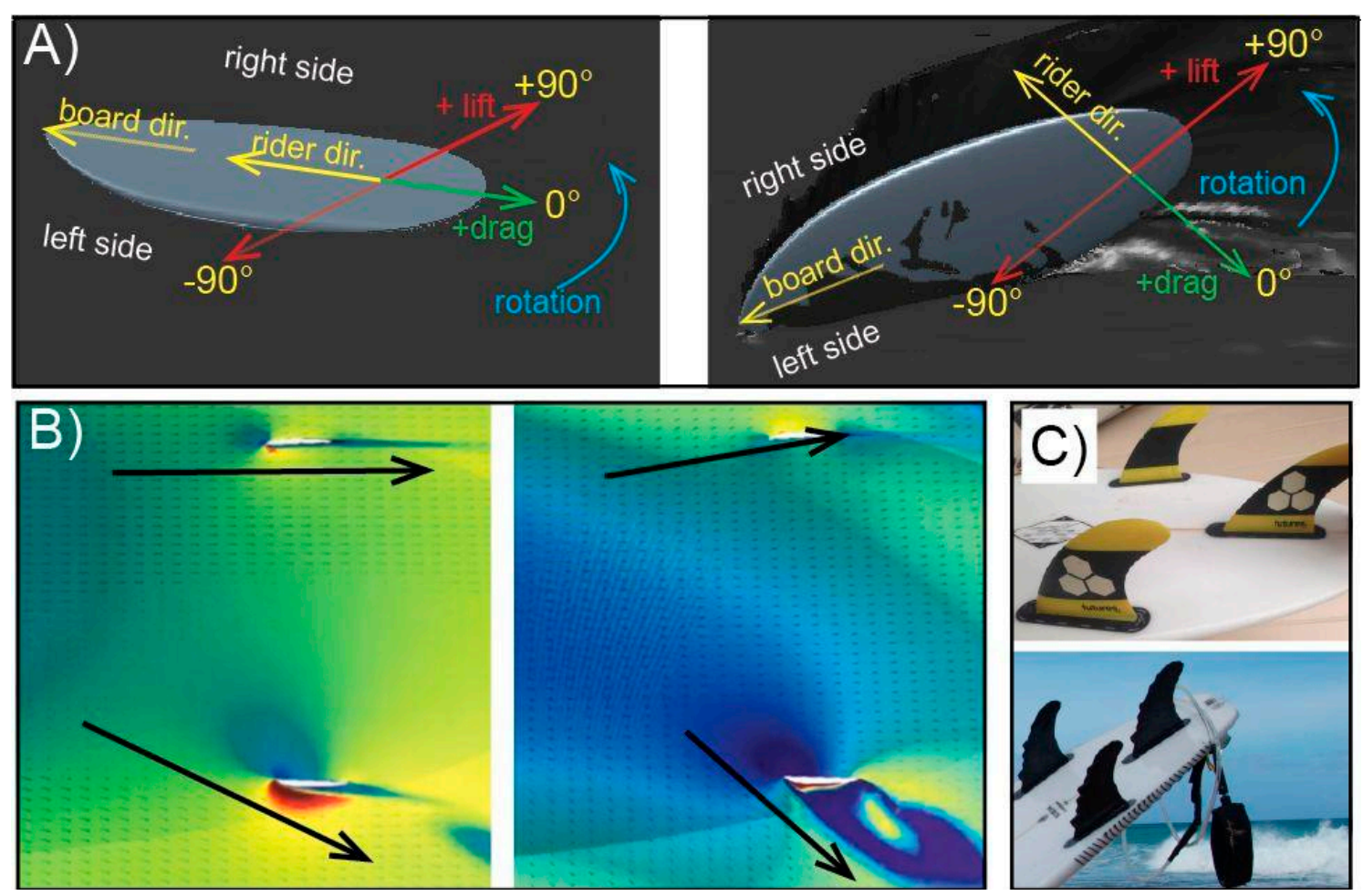

Figure 1. (A) Free body diagram of lift and drag forces experienced by the rider from the fins. On the left is prior to turning, right is at the end of the turn. During rotation, rider direction is tangent to the turn. (B) Flow directions under the surfboard in the middle (left) and end (right) of a turn. This example is of a twin fin configuration not used in this study. (C) Examples of control (top) and humpback whale-inspired fin (RW) (bottom) thruster combinations. See Figure S2 for thruster positioning and angles used for simulations.

\subsection{CFD Research}

Dynamic CFD methods using STAR-CCM+ are similar to those detailed in References [8-10], although [10] did not consider the air/water interface as this study does. Field performance means 
in Table S2 were used to generate the speeds and rotation rates used in CFD simulations (Table 1). Field results from surfing ocean waves showed rotation rates increased with skill level, while the surfer's speed during the cutback was more of a function of wave energy. Therefore, flow speed remained constant at $7 \mathrm{~m} / \mathrm{s}$ for each skill level. The time step between simulations was $0.002 \mathrm{~s}$, which resulted in 400 simulations per treatment, run from 0.0 to $0.8 \mathrm{~s}$. A total of 6 simulations were run (2 fin types $\times 3$ treatments).

Table 1. Matrix of speeds and rotation rates used in CFD simulations. Figure S1 describes roll, pitch and yaw orientation in more detail.

\begin{tabular}{ccccc}
\hline $\begin{array}{c}\text { Skill Level Per } \\
\text { [7] }\end{array}$ & $\begin{array}{c}\text { Flow Speed } \\
(\mathbf{m} / \mathbf{s})\end{array}$ & $\begin{array}{c}\text { Roll Rate } \\
(\mathbf{r a d} / \mathbf{s})\end{array}$ & $\begin{array}{c}\text { Pitch Rate } \\
(\mathbf{r a d} / \mathbf{s})\end{array}$ & $\begin{array}{c}\text { Yaw Rate } \\
(\mathbf{r a d} / \mathbf{s})\end{array}$ \\
\hline Intermediate & 7.0 & 1.00 & 0.50 & 2.25 \\
Expert & 7.0 & 1.25 & 0.75 & 2.75 \\
WCT & 7.0 & 1.5 & 1.00 & 3.25 \\
\hline
\end{tabular}

\subsubsection{Force analysis}

Because of the complexities of analyzing motion on all three axes, traditional approaches of analyzing individual lift and drag proved to add to the complexity. On top of that, the frame of reference is important, as the board direction and rider direction (forces rider feels) can vary significantly during a turn (Figure 1A). Flow direction relative to each fin can also vary significantly (Figure 1B). We simplified analysis by calculating a single resultant magnitude (force) and direction based on fin lift and drag forces in the rider direction.

Figure 1A details rider direction. At the start of a simulation, board and rider direction are the same. Increased lift (+Lift) is assigned in the direction of the turn, which in our simulations is towards the right fin, making the left fin more of the "pivot point" of the turn. Once the board starts turning, rider and board direction diverge. Rider direction becomes tangent to the turn, while board direction remains in the direction of the board.

\section{Results and Discussion}

\subsection{Field Research}

Table 2 lists all performance data. Table S3 provides a description of each measurement. For turn performance (not speeds), all 10 performance means are higher for RW fins, with 70\% significantly higher. Surfers also felt better control of the surfboard when using RW fins, including an improved ability to complete a turn and transition into the next maneuver.

Table 2. Control vs. RW field performance means. Table S3 lists descriptions of each measurement.

\begin{tabular}{cccc}
\hline Fieldwork Summary & Control Fins & RW Fins & $p$-Value \\
\hline \# cutbacks & 815 & 666 & - \\
Mean session speed (m/s) & $5.3 \pm 0.1$ & $5.1 \pm 0.1$ & $\mathbf{0 . 0 0 5}$ \\
Bottom turn initial speed (m/s) & $7.1 \pm 0.1$ & $7.1 \pm 0.1$ & 0.512 \\
Cutback speed (m/s) & $6.4 \pm 0.1$ & $6.3 \pm 0.1$ & 0.873 \\
\hline Performance Means & & \\
\hline yaw rate $(\mathrm{rad} / \mathrm{s})$ & $2.6 \pm 0.1$ & $2.7 \pm 0.1$ & 0.143 \\
roll rate $(\mathrm{rad} / \mathrm{s})$ & $1.1 \pm 0.04$ & $1.1 \pm 0.05$ & 0.094 \\
pitch rate $(\mathrm{rad} / \mathrm{s})$ & $0.7 \pm 0.03$ & $0.7 \pm 0.04$ & $\mathbf{0 . 0 0 5}$ \\
yaw power $(\mathrm{W})$ & $140 \pm 14$ & $163 \pm 17$ & $\mathbf{0 . 0 3 5}$ \\
roll power $(\mathrm{W})$ & $28 \pm 3$ & $36 \pm 5$ & $\mathbf{0 . 0 1 1}$ \\
pitch power $(\mathrm{W})$ & $13 \pm 2$ & $18 \pm 4$ & $\mathbf{0 . 0 0 5}$
\end{tabular}




\begin{tabular}{cccc} 
Total power $(\mathrm{W})$ & $180 \pm 18$ & $216 \pm 24$ & $\mathbf{0 . 0 1 9}$ \\
Total power/Inertia & $6.6 \pm 0.6$ & $7.6 \pm 0.8$ & $\mathbf{0 . 0 4 3}$ \\
$\mathrm{C}_{\mathrm{p}}$ & $0.11 \pm 0.01$ & $0.12 \pm 0.01$ & $\mathbf{0 . 0 4 2}$ \\
Trace cutback power & $4.0 \pm 0.2$ & $4.0 \pm 0.2$ & 0.625 \\
\hline
\end{tabular}

\subsection{CFD Research}

\subsubsection{Skill Level Comparison}

Tables 3-5 display mean rider direction resultant force magnitudes and angles relative to rider direction for each fin. Figure 2 shows the resultant forces versus time. Because these are resultant forces in the rider direction, it is important to note the values represent the amount of sustained force, i.e., impulse (force $\bullet$ time) a surfer must impart in order to perform the maneuver.

Resultant means were calculated during the cutback, starting at $0.25 \mathrm{~s}$ into a simulation, and ending at $0.8 \mathrm{~s}$ for intermediate, $0.7 \mathrm{~s}$ for expert, and $0.6 \mathrm{~s}$ for WCT. Higher skill levels took less time to complete the turn because the rotation rates were faster. Video analysis of each treatment is found in Videos S1 to S6, which show color-mapped pressures on each fin during the cutback simulation, and air/water interactions beneath the surfboard.

Table 3. Intermediate (skill Level 6) mean Resultant forces and directions, relative to rider direction during cutback, from 0.25 to $0.8 \mathrm{~s}$. Significant $p$-values in bold.

\begin{tabular}{ccccccc}
\hline Intermediate & \multicolumn{3}{c}{ Resultant Magnitude (N) } & \multicolumn{3}{c}{ Direction (deg) } \\
\hline & Control & RW & $p$-value & Control & RW & $p$-value \\
Left fin & $194 \pm 8$ & $184 \pm 8$ & 0.116 & $-69 \pm 2$ & $-69 \pm 2$ & 0.925 \\
Center fin & $209 \pm 9$ & $212 \pm 9$ & 0.635 & $-65 \pm 2$ & $-65 \pm 2$ & 0.960 \\
Right fin & $155 \pm 6$ & $179 \pm 8$ & $<0.001$ & $-55 \pm 2$ & $-58 \pm 2$ & $\mathbf{0 . 0 2 9}$ \\
All fins & $554 \pm 23$ & $574 \pm 25$ & 0.250 & $-64 \pm 2$ & $-64 \pm 2$ & 0.715 \\
\hline
\end{tabular}

Table 4. Expert (skill Level 7/8) mean Resultant forces and directions, relative to rider direction during cutback, from 0.25 to $0.7 \mathrm{~s}$. Significant $p$-values in bold.

\begin{tabular}{ccccccc}
\hline Expert & \multicolumn{3}{c}{ Resultant Magnitude (N) } & \multicolumn{3}{c}{ Direction (deg) } \\
\hline & Control & RW & $p$-value & Control & RW & $p$-value \\
Left fin & $213 \pm 10$ & $203 \pm 10$ & 0.186 & $-70 \pm 2$ & $-70 \pm 2$ & 0.960 \\
Center fin & $225 \pm 11$ & $225 \pm 10$ & 0.974 & $-66 \pm 2$ & $-66 \pm 2$ & 0.959 \\
Right fin & $168 \pm 8$ & $189 \pm 9$ & $<0.001$ & $-57 \pm 2$ & $-60 \pm 2$ & $\mathbf{0 . 0 4 5}$ \\
All fins & $605 \pm 28$ & $616 \pm 29$ & 0.542 & $-65 \pm 2$ & $-66 \pm 2$ & 0.708 \\
\hline
\end{tabular}

Table 5. WCT (skill Level 9) mean Resultant forces and directions, relative to rider direction during cutback, from 0.25 to $0.6 \mathrm{~s}$. Significant $p$-values in bold.

\begin{tabular}{ccccccc}
\hline WCT & \multicolumn{3}{c}{ Resultant Magnitude (N) } & \multicolumn{3}{c}{ Direction (deg) } \\
\hline & Control & RW & $p$-value & Control & RW & $p$-value \\
Left fin & $234 \pm 13$ & $219 \pm 12$ & 0.084 & $-74 \pm 2$ & $-74 \pm 2$ & 0.904 \\
Center fin & $249 \pm 14$ & $244 \pm 13$ & 0.572 & $-70 \pm 2$ & $-70 \pm 2$ & 0.931 \\
Right fin & $174 \pm 10$ & $189 \pm 11$ & $\mathbf{0 . 0 4 5}$ & $-60 \pm 2$ & $-63 \pm 2$ & 0.057 \\
All fins & $654 \pm 36$ & $650 \pm 36$ & 0.872 & $-69 \pm 2$ & $-69 \pm 2$ & 0.799 \\
\hline
\end{tabular}

Comparing RW and $C$ by skill level in Tables $3-5$ and Figure 2, reveals that as rotation rate (i.e., skill level) increases, a greater impulse is required from the surfer to complete the turn. Field results in Table S2 follow this pattern, with higher skill level surfers generating more power. Also, the majority of the forces are imparted on the left and center fin, with a negative direction against 
the rider. Falk et al. [10] noted this direction produces a turning moment that realigns rider and board direction and stabilizes the surfboard. Tables 3-5 show that as turn rate increases, the direction becomes more negative. In other words, not only is a greater impulse required to complete a higher skill level turn, it is more directly opposing the rider, requiring even more surfer power input.

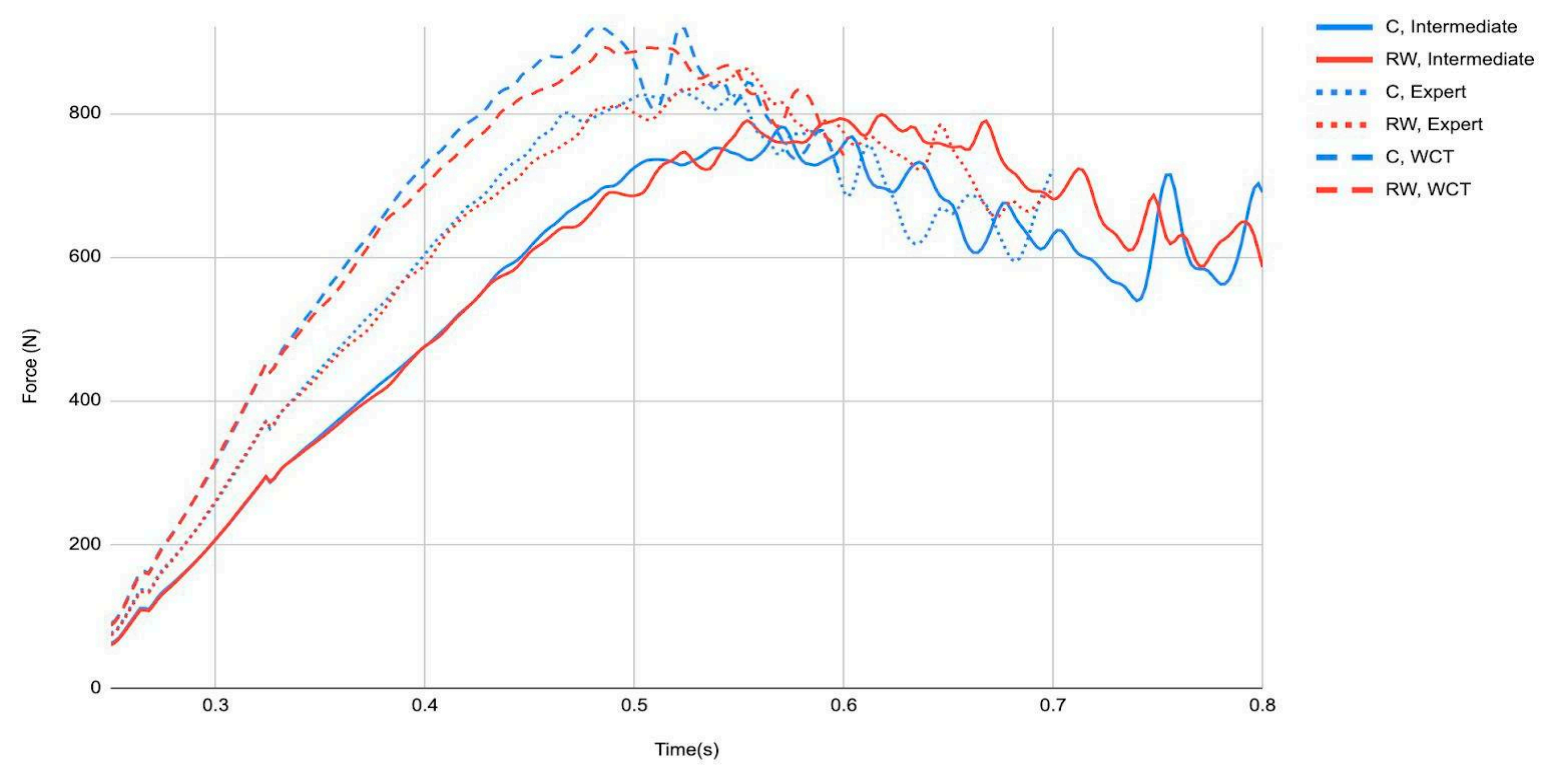

Figure 2. Time series of rider direction resultant forces during the turn. All turns start at $0.25 \mathrm{~s}$, with intermediate/expert/WCT ending at 0.8/0.7/0.6 s, respectively.

\subsubsection{Control vs. RW}

Most of the differences in Tables 3-5 are not significant. As evident in Figure 2 and Videos S1 to S6, the center and right fins encounter large amounts of turbulence towards the end of each turn, which makes it more difficult to evaluate differences. Note that our turns were generated with a constant $7 \mathrm{~m} / \mathrm{s}$ flow impacting the fins. In a real turn, the speed usually decreases as the turn progresses. Therefore, turbulence would be expected in a real turn, but perhaps to a lesser degree than our simulation results.

Nevertheless, some consistent patterns are found in Tables 3-5. For the left fins, RW's resultant is 5.2-6.4\% less. For the turns shown in Videos S1 to S6, the left fin is an important pivot point, so having a lower resultant can improve maneuverability as others have also suggested [11]. Figure 2 also shows that, at the start of each turn, RW's resultant is consistently lower, which again could help make it more maneuverable. Moreover, note that in Figure 2 RW exhibits patterns consistent with delayed stall and gradual stall observed in other tubercled designs [12]. These patterns may benefit the surfer because there is a more constant force to respond to during the latter half of the turn.

The only significant differences in Tables $3-5$ occur for the right fins, where RW is consistently higher for both the resultant and direction. The right fin receives a large amount of turbulence coming off the side, or rail of the surfboard. These results suggest that RW's passive flow control is more capable of handling turbulent flow.

Table 6 further breaks down a WCT-level. Before the turn, resultant forces are low and mostly due to drag, and only differ by $1.6 \%$. During the turn, RW is lower, again suggesting improved maneuverability, with a $2.8 \%$ difference. After the turn, when the surfer is regaining control to initiate the next maneuver, notice the significant difference in forces, but also confidence intervals, which differ by $10.5 \%$. Clearly, the RW fins are providing a damping effect to turbulent flow, which is a conclusion that others have also reached $[12,13]$. Perhaps most importantly, participants felt this improved post-turn stability of RW fins. 
Table 6. Mean resultants for before (0.022 to $0.222 \mathrm{~s})$, during ( 0.3 to $0.5 \mathrm{~s}$ ) and after ( 0.6 to $0.8 \mathrm{~s})$ a WCT-level turn. Confidence levels for After rounded to 1 decimal place to show detail.

\begin{tabular}{cccc}
\hline WCT & & \multicolumn{2}{c}{ All Fins Resultant (N) } \\
\hline & Control & RW & $p$-value \\
Before & $12.3 \pm 0.09$ & $12.5 \pm 0.05$ & $<\mathbf{0 . 0 0 1}$ \\
During & $690 \pm 36$ & $671 \pm 33$ & 0.431 \\
After & $733 \pm 7.6$ & $755 \pm 6.8$ & $<\mathbf{0 . 0 0 1}$ \\
\hline
\end{tabular}

\section{Conclusions}

Traditionally, surfing performance is highly subjective, often based on opinion with little or no quantifiable support. In this paper, we have demonstrated that comparing field and numerical results of shortboard surfing performance yield powerful insights. For example, compared to an intermediate level surfer, a WCT-level surfer must impart, on average about 18\% more sustained force to complete a WCT-level turn using Control fins, but only 13\% more using RW fins.

Comparing Table S1 WCT (skill Level 9) data to a skill Level 8 surfer using control fins, only $20 \%$ of the Table 2 performance data shows no significant difference, with the remaining $80 \%$ significantly lower for skill Level 8. However, using RW fins, 90\% of Table 2 performance data shows no significant difference to the WCT data. The reduced force required to turn RW fins may have allowed surfers to turn at faster rates and generate significantly more power in ocean surfing conditions, i.e., enabling an expert surfer to surf more like a WCT-level surfer when using RW fins. The improved stability of RW fins, both evidenced in the CFD results and felt by surfers may also result in more efficient power transfer into the turn.

Supplementary Materials: The following are available online at https://surfengineers.com/isea2020a, Figure S1: Bottom turn and cutback maneuvers, Figure S2: Thruster fin positioning used in simulations, Table S1: Surfer, surfboard, and surfing fin specifications, Table S2: Field performance means versus skill level, Table S3: Description of performance measurements listed in Table 2 and Table S2, Video S1: Intermediate C.mp4, Video S2: Intermediate RW.mp4, Video S3: Expert C.mp4, Video S4: Expert RW.mp4, Video S5: WCT C.mp4, Video S6: WCT RW.mp4.

Acknowledgments: Special thanks to Josiah Swanson for CAD prep, and trial-and-error RW prototype testing. Field research funding provided by DIVE, LLC (diveintomath.com). MihP would like to thank the Global Challenges Program at the University of Wollongong, Australia, for financial assistance. We thank J. Barthelemy and M. Amirghasemi for technical assistance with accessing the Trace API.

Conflicts of Interest: The authors declare no conflict of interest.

\section{References}

1. Shormann, D.E.; in het Panhuis, M. Performance evaluation of a humpback whale-inspired hydrofoil design applied to surfboard fins. In Proceedings of the OCEANS 2019 MTS/IEEE Conference, Seattle, WA, USA, 27-31 October 2019.

2. Warshaw, M. The History of Surfing; Chronicle Books: San Francisco, CA, USA, 2010.

3. Farley, O.R.L. Competitive Surfing: A Physiological Profile of Athletes and Determinants of Performance. Master's Thesis, Auckland University of Technology, Auckland, Australia, 2011.

4. Farley, O.R.L.; Abbiss, C.R.; Sheppard, J.M. Performance Analysis of Surfing: A Review. J. Strength Cond. Res. 2017, 31, 260-271, doi:10.1519/JSC.0000000000001442.

5. Gately, R.D.; Beirne, S.; Latimer, G.; Shirlaw, M.; Kosasih, B.; Warren, A.; Steele, J.R.; in het Panhuis, M. Additive Manufacturing, Modeling and Performance Evaluation of 3D Printed Fins for Surfboards. Mrs Adv. 2017, 2, 913-920, doi:10.1557/adv.2017.107.

6. Shormann D.E.; in het Panhuis, M. Performance evaluation of humpback whale-inspired shortboard surfing fins based on ocean wave fieldwork. PLoS ONE 2020 15, e0232035.

7. Hutt, J.A.; Black, K.P.; Mead, S.T. Classification of Surf Breaks in Relation to Surfing Skill. J. Coast. Res. 2001, 29, 66-81. 
8. Oggiano, L. Numerical Comparison between a Modern Surfboard and an Alaia Board using Computational Fluid Dynamics (CFD). In Proceedings of the 5th International Congress on Sport Sciences Research and Technology Support (icSPORTS 2017), Madeira, Portugal, 30-31 October 2017; pp. 75-82.

9. Oggiano, L.; Pierella, F. CFD for surfboards: Comparison between three different designs in static and maneuvering conditions. In Proceedings of the 12th Conference of the International Sports Engineering Association, Brisbane, Queensland, Australia, 26-28 March 2018.

10. Falk, S.; Kniesburges, R.; Janka, R.; Grosso, R.; Becker, S.; Semmler, M.; Dollinger, M. Computational hydrodynamics of a typical 3-fin surfboard setup. J. Fluids Struct. 2019, 90, 297-314.

11. El-Atm, B.; Kelson, N.; Gudimetla, P. A finite element analysis of the hydrodynamic performance of 3and 4-fin surfboard configurations. In Proceedings of the 9th Global Congress on Manufacturing and Management (GCMM2008), Surfers Paradise, Australia, 12-14 November 2008.

12. Aftab, S.M.A.; Razak, N.A.; Mohd, Rafie, A.S.; Ahmad, K.A. Mimicking the humpback whale: An aerodynamic perspective. Prog. Aerosp. Sci. 2016, 84, 48-69, doi:10.1016/j.paerosci.2016.03.002.

13. Tong, F.; Qiao, W.; Chen, W.; Cheng, H.; Wei, R.; Wang, X. Numerical analysis of broadband noise reduction with wavy leading edge. Chin. J. Aeronaut. 2018, 31, 1489-1505, doi:10.1016/j.cja.2018.03.020.

(C) 2020 by the authors. Licensee MDPI, Basel, Switzerland. This article is an open access article distributed under the terms and conditions of the Creative Commons Attribution (CC BY) license (http://creativecommons.org/licenses/by/4.0/). 